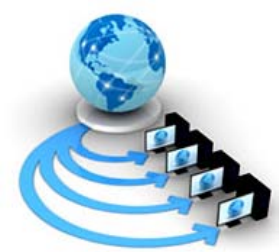

Volume 9, No. 1, January-February 2018

\title{
A SURVEY OF FUZZY C-MEANS BASED ALGORITHMS FOR SEGMENTATION OF BRAIN MAGNETIC RESONANCE IMAGES
}

\author{
Nighat Nazir \\ Student of M.Tech (CSE) \\ Department of Computer Science, \\ Punjabi University, India
}

\author{
Aamir Hamid Lone \\ Student of M.Tech (CSE) \\ Department of Computer Science and Engineering, \\ Al-Falah University Faridabad Haryana, India
}

\begin{abstract}
Segmentation of Brain tissues have aim to segment different tissues like cerebrospinal fluid (CSF) from magnetic resonance images (MRI), gray matter (GM) and white matter (WM). In the past, many researchers have proposed MRI segmentation techniques in the field of medical image and soft computing. A number of segmentation techniques have been proposed among them thresholding, region-based segmentation, classificationbased segmentation are the mostly used technique. In this paper, a comprehensive overview of fuzzy C-means (FCM) based algorithms for MRI segmentation is presented.
\end{abstract}

Keywords: Magnetic Resonance Image (MRI), Segmentation, Fuzzy-C means (FCM).

\section{INTRODUCTION}

Image Segmentationis one of the important tasks in digital image processing. It is defined as a process of dividing an input image into number of regions. In Bio-Medical Image Segmentation has great and vital significance. In case of brain tumor, segmentation consists of segmenting the tumor tissues such as active cells, necrotic cells, edema from normal tissues such as white matter (WM), gray matter (GM), and cerebrospinal fluid (CSF). Manual segmentation of medical resonance images (MRI) requires software tools with graphical user interface for drawing the regions of interest. Manual Segmentation is a time consuming process. MRI scanners generate multiple two-dimensional cross-sections, and human expert has to go through the data set slice by slice for choosing the most representatives ones from which the relevant regions are carefully delineated. So there is a need of automated delineation of different tissues in brain. There are various brain imaging techniques like computed tomography (CT), positron emission tomography (PET) and magnetic resonance images (MRI) that can provide information about the brain tissues. Anatomy of brain can be scanned by MRI and CT scan but MRI is better than CT scan for diagnosis of diseases. MRI does not affect the human body because it does not use any radiation. Various MRI sequences have been employed for diagnosis in current medical routines. These sequences include T1-weighted MRI (T1w), T1- weighted MRI with contrast enhancement (T1wc), Proton Densityweighted MRI (PDw), and Fluid-Attenuated Inversion Recovery (FLAIR).T1w sequence images makes an easy annotation of healthy tissues while, T1wc sequence images brightens the border areas of the brain tumor by accumulating the contrast agents at the border. FLAIR is regarded as a highly effective sequence image to distinguish the edema region from CSF. In the last years, many researchers in the field of medical imaging and soft computing have made significant advances in the field of brain MRI segmentation

\section{II.PREPROCESSING OF MRI}

MRI is often corrupted with noise components that can lead to deviation from the desired results during the various image processing steps, including segmentation. These need to be removed using filters. In order to improve the accuracy of segmentation from the image, pre-processing of MRI has been introduced. Brain images are more sensitive than other medical images; therefore they should have minimum noise and maximum quality. The preprocessing operations include image denoising and skull-stripping.

\section{A. Denoising}

MRI are prone to noises, particularly Rician noise. The presence of noise makes it difficult to distinguish the regions of interest, such as the tumor tissues from the normal brain tissues. Various denoising filters are employed in order to remove noise and thus enhance the contrast between the various regions of the image. These include local smoothing filters, such as anisotropic diffusion filter (ADF) [1], neighboring filter [1] and non local means filter (NL-means) [1]. Local smoothing filters are average filters that perform averaging locally whereas NL-means filters are based on non local averaging of all pixels in the image. The main drawback of local smoothing filters is that these filters compare only the grey level values of a single pixel. The robustness of these filters is lowered if the grey level values of the image pixel are noisy. On the other hand NL-means filters not only compare 
the grey level values, but also the geometrical configuration of the entire neighborhood. Hence, NL-means filters are strong and robust than local smoothing filters.

\section{B. Skull-Stripping}

Skull stripping is an important preprocessing step for the analysis of MRI. The background of an image does not contain any information of interest but increases the processing time. Therefore there is need to remove the background and other unnecessary regions. Various Skullstripping tools have been used for this purpose like brain extraction tool (BET) [2], the brain surface extractor (BSE) [3], and the hybrid watershed (HWA) [4].

\section{BRAIN SEGMENTATION METHODS OF MRI}

Different method have been employed for segmentation of MRI. Among them thresholding [5], region-based segmentation [6], classification-based segmentation are most widely used.

\section{A. Thresholding Segmentation}

Thresholding [5] is one of the simplest technique used for segmentation of MRI. Thresholding divides an MRI into various regions by comparing the each pixel value with a predefined threshold value. Thresholding can be either local or global. One of the limitation of thresholding is that it only considers the intensity, not any relationship between the pixels. Global thresholding works well if the MRI contains the homogenous regions or the contrast between the dark background and light pixel is very high. However, it may fail if the two or more regions have overlapping intensities. If the contrast between the MRI is low then, selection threshold value becomes more difficult. In local thresholding, threshold value is determined in a local region around a pixel. Local thresholding values can be determined by statistical properties such as mean intensity value in T1-weighted MRI.

\section{B. Region-Based Segmentation}

In MRI, region-based segmentation [6] is used to group the pixels with similar properties. The first requirement is to select the seed point and then form the regions by appending neighboring pixel according to the specific criteria. The advantage of this method is that it can easily separate the regions with similar property and generate connected region. However, this method involves the manual selection of selection of seed, which is the time consuming process.

\section{Classification Segmentation}

In classification based segmentation, fuzzy C-means (FCM) [7] algorithm is widely used for segmentation of MRI as it can preserve more information than hard segmentation methods. FCM is a clustering algorithm developed by Dunn [8], and later extended by Bezdek [9] is an improvement over Kmeans. In k-means [10] which allows pixel belong to single cluster. The main limitation of K-means method is that the final result is dependent on the initial centroid value to great extent. While in FCM [7 It permits pixel belong to multiple clusters having variations in degree of membership value. The main drawback of FCM [7] is that it does not consider the any information of spatial domain, as it is very sensitive to noise. Because medical images are corrupted by Rician noise and intensity inhomogeneity caused by operator performance, equipment and the environment, the segmentation with FCM becomes problematic. So, in order to incorporate the spatial information into standard FCM many researchers proposed various variants of FCM. Ahmed et al [11] proposed fuzzy Cmeans with spatial constraint (FCM_S) altered the objective function of standard FCM in order to incorporate spatial information. Disadvantage of FCM_S is increase in computational time because iteration steps are used to compute neighboring terms. In FCM_S, MRI segmentation depends mainly upon size of image, as larger size of image is it will take more time for segmentation. So in order to reduce computational time chen and zang[12] developed two variants of FCM. One is called FCM_S1 and another variant as FCM_S2.Replacing of neighboring terms of FCM_S,Both FCM_S1 and FCM_S2 computes mean and median filtered image in advance. Both FCM_S, FCM_S1 and FCM_S2 are dependent on regularization parameter $(\alpha)$ so that it controls tradeoff between original and corresponding median filtered image and mean.

. However, if the value of regularization parameter $(\alpha)$ is equal to 0, then algorithm works as the standard FCM. Cai et al. [13] proposed the fast generalized fuzzy C-means algorithm (FGFCM) that not only reduces the limitation of FCM_S but also improves the clustering performance. FGFCM introduces the two factors introduces both local spatial information and local gray information function to generate a new image equation as:

$$
=\frac{\sum_{\epsilon}}{\sum_{\epsilon}}
$$

where represents the $j$ thpixel of an image, represents the similarity measure between $j$ th pixel and its neighboring rth pixels, represents the neighboring pixels around the $j t h$ pixel. Fuzzy local information C-means (FLICM) [14] uses both local spatial information and local gray information in order to guarantee noise insensitiveness and preserve the image details. The main advantage of FLICM is independent of any regularization parameter unlike in FCM_S, FCM_S1, FCM_S2 and FGFCM. Adhikari et al. [15] proposed conditional spatial fuzzy C-means algorithm (csFCM) incorporated both local spatial membership value and global spatial membership value in order to reduce the problems of sensitivity to noise and intensity inhomogeneity in MRI.

\section{COMPARISON PARAMETERS}

In order to compare the performance of various fuzzy Cmeans based methods, various MRI segmentation techniques are analyzes using different comparison parameters like

\section{A. Dice coefficient}

Dice coefficient [16] are used for evaluating the accuracy of segmentation technique. For a given image if and represents the set pixels belong to segmented output and its 
corresponding ground truth image. Output of segmented image is compared with its corresponding values of ground truth image its resemblance with other each cluster is calculated as :

$$
=2 \times \frac{\cap}{+}
$$

\section{B. Jaccard similarity}

Jaccard similarity [16] is defined as ration between intersection and union of segmented class and ground truth class Jaccard similarity is given by:

$$
=\frac{\cap}{U}
$$

Dice coefficient and Jaccard similarity value ranges between 0 and 1 , with value near to 0 indicates lesser amount of common pixels between segmented image and its corresponding ground truth image/ the value near to 1 indicates more accurate segmentation.

\section{CONCLUSION}

FCM is one of the most widely used in many application domains. This paper provides a comprehensive overview of various FCM-based methods for segmentation of MRI. This paper presents advantages and disadvantages of various FCMbased methods.

\section{REFERENCES}

[1] Buades, Antoni, BartomeuColl, and J-M. Morel. "A non-local algorithm for image denoising." 2005 IEEE Computer Society Conference on Computer Vision and Pattern Recognition (CVPR'05). Vol. 2. IEEE, 2005.

[2] Smith, Stephen M. "Fast robust automated brain extraction." Human brain mapping 17.3 (2002) 143-155.

[3] Shattuck, David W., and Richard M. Leahy. "BrainSuite: An automated cortical surface identification tool." International Conference on Medical Image Computing and ComputerAssisted Intervention. Springer Berlin Heidelberg, 2000.
[4] Ségonne, Florent, et al. "A hybrid approach to the skull stripping problem in MRI." Neuroimage 22.3 (2004) 10601075.

[5] Sujji, G. Evelin, Y. V. S. Lakshmi, and G. WiselinJiji. "MRI brain image segmentation based on thresholding." International Journal of Advanced Computer Research 3.1 (2013) 97-101.

[6] Dubey, R. B., M. Hanmandlu, and S. K. Gupta. "Region growing for MRI brain tumor volume analysis." Indian Journal of Science and Technology 2.9 (2009) 26-31.

[7] Agrawal, Sanjay, Rutuparna Panda, and Lingraj Dora. "A study on fuzzy clustering for magnetic resonance brain image segmentation using soft computing approaches." Applied Soft Computing 24 (2014): 522-533.

Dunn, Joseph C. "A fuzzy relative of the ISODATA process and its use in detecting compact well-separated clusters." (1973) 32-57.

[9] Bezdek, James C. Pattern recognition with fuzzy objective function algorithms. Springer Science \& Business Media, 2013.

[10] Agrawal, Sanjay, Rutuparna Panda, and Lingraj Dora. "A study on fuzzy clustering for magnetic resonance brain image segmentation using soft computing approaches." Applied Soft Computing 24 (2014) 522-533.

[11] Ahmed, Mohamed N., et al. "A modified fuzzy c-means algorithm for bias field estimation and segmentation of MRI data." IEEE transactions on medical imaging 21.3 (2002) 193-199

[12] Chen, Songcan, and Daoqiang Zhang. "Robust image segmentation using FCM with spatial constraints based on new kernel-induced distance measure." IEEE Transactions on Systems, Man, and

Cybernetics, Part B (Cybernetics) 34.4 (2004) 1907-1916.

[13] Cai, Weiling, Songcan Chen, and Daoqiang Zhang. "Fast and robust fuzzy c-means clustering algorithms incorporating local information for image segmentation." Pattern Recognition 40.3 (2007) 825-838

[14] Krinidis, Stelios, and VassiliosChatzis. "A robust fuzzy local information C-means clustering algorithm." IEEE Transactions on Image Processing 19.5 (2010) 1328-1337.

[15] Adhikari, Sudip Kumar, et al. "Conditional spatial fuzzy Cmeans clustering algorithm for segmentation of MRI images." Applied soft computing 34 (2015) 758-769

[16] Agrawal, Sanjay, Rutuparna Panda, and Lingraj Dora. "A study on fuzzy clustering for magnetic resonance brain image segmentation using soft computing approaches." Applied Soft Computing 24 (2014) 522-533. 